\title{
Computed tomogram guided fine-needle aspiration cytology of lung mass with histological correlation: A study in Eastern India
}

\author{
Santosh Kumar Mondal, Dipanwita Nag, Rama Das, Palash Kumar Mandal, Pranab Kr Biswas, Manish Osta
}

\section{Abstract}

Background: Fine-needle aspiration cytology (FNAC) is an important and useful investigation, and is considered next to imaging in the rapid diagnosis of pulmonary mass lesion for the last few decades. Aims: To assess the role of Computed Tomogram (CT) guided FNAC in pulmonary mass lesions; to analyze the results; and to compare with histopathological findings. Materials and Methods: The clinical, radiological, and cytological data of I 30 patients were prospectively studied who underwent CT guided FNAC from October 2009 through September 20I I. Thereafter these patients underwent bronchoscopic/trucut biopsy/ lobectomy, whatever clinically indicated. Smears and tissue sections were evaluated simultaneously to reach at a definite diagnosis. Results: Out of 130 cases, we found adequate FNAC smear and histopathology reports only in 124 cases. The age range varied from 35 to 73 years with the peak in the fifth to sixth decades. The benign lesions were $10(8.07 \%)$ and malignant lesions were I I4 $(91.93 \%)$ shown by cytology. The most common tumor was adenocarcinoma $(51.72 \%)$ followed by squamous cell carcinoma (22.41\%) and small cell carcinoma 6.89\%. Diagnostic accuracy of CT guided FNAC was $95 \%$. Post procedural complications such as hemorrhage and chest pain were minimal and were noted only in three cases. Conclusion: CT guided FNAC of pulmonary masses provides simple, easy, and reliable method for reaching rapid tissue diagnosis with minimal complication.

Key words: FNAC, histological correlation, pulmonary mass

\section{Introduction}

Fine-needle aspiration cytology (FNAC) is a simple, relatively safe, rapid, reliable technique for the diagnosis of pulmonary mass lesions, particularly with the aid of computed tomography (CT) scan. FNAC not only distinguishes between benign and malignant lesions but also helps in tumor typing of lung cancer, so initiation of specific therapy like chemotherapy or surgery is possible without unnecessary delay.

FNAC was first used by Martin and Ellis ${ }^{[1]}$ as a diagnostic tool. Leyden in 1883 and Menbriel in 1986 introduced the technique as diagnostic lung puncture for detection of malignancy and infections. ${ }^{[2]}$ More than three decades have passed, FNAC of pulmonary mass has gained worldwide momentum and acceptance, as it has further enhanced the diagnostic usefulness of clinical cytology in lung and mediastinal cancer diagnosis. ${ }^{[3]}$ FNAC has long been used for the non-surgical confirmation of primary as well as metastatic thoracic lesions. The special advantage of FNAC includes detection of those tumor types like small cell carcinoma, lymphomas more appropriately

Department of Pathology, Medical College, Kolkata, West Bengal, India Correspondence to: Dr. Santosh Kumar Mondal,

E-mail:dr_santoshkumar@hotmail.com

\begin{tabular}{|l|l|}
\hline \multicolumn{2}{|c|}{ Access this article online } \\
\hline Quick Response Code: & Website: \\
\hline & www.sajc.org \\
\cline { 2 - 2 } & \\
\hline
\end{tabular}

treated by chemotherapy rather than surgery. Numerous literatures supported that CT-guided FNAC is an accurate and sensitive way of diagnosing malignancy of the thorax. ${ }^{[4,5]}$ This procedure is almost minimum painful nonoperative procedure as compared with biopsy for diagnosis of pulmonary mass, outweighs the single major rare complication of pneumothorax. ${ }^{[5]}$

The present study was undertaken to know the pathological spectrum of pulmonary lesions; to correlate cytological findings with histological features; and to assess the accuracy of FNAC in evaluation of lung masses.

\section{Materials and Methods}

The study was conducted in the department of Pathology, in our medical college and hospital, during the period of Sep 2009 - Aug 2011. There were consecutive cases over a period of two years. One hundred and thirty (130) patients having pulmonary mass lesions suspected to be neoplastic by chest radiograph and/or CT scan were referred from different departments like chest, medicine, and oncology. One hundred and twenty four (124) cases were included in this prospective study as cytological examination showed an inadequate cell in six cases. This prospective study was approved by the hospital ethics committee.

CT guided FNAC of pulmonary mass lesions from each case were performed by our senior pathologist as OPD procedure and also in presence of experienced radiologist after explaining the risks and benefits. From each patient, informed consent was taken. The skin surface was cleaned with povidone iodine, and then $21 \mathrm{G}-88 \mathrm{~mm}$ long spinal needle was introduced through percutaneous/transthoracic approach localizing the exact position by CT scan after the measurement of the site and angle of entry of the needle, 
route of the needle, and the distance between the skin and lesion on the CT scan monitor. ${ }^{[6]}$ Following placement of the needle, a CT scan slice was taken to ascertain whether the tip of the needle was within the mass. The aspirate was obtained by to and fro and rotating movements of the needle within the lesions and five smears were prepared immediately from the sample in the CT scan room. Air-dried smears were stained with Leishman-Giemsa (L and G) stain, whereas alcohol-fixed smears were stained with Papanicolaou (PAP) stain for rapid cytopathological evaluation of the lesions.

Patients were kept for 2 hours under observation. In our study, mild hemorrhage from peri-lesional area in one case and chest pain in two cases was found, which could be managed conservatively. We exclude those cases that were suffering from severe

Chronic obstructive pulmonary disease (COPD), bleeding disorders, pulmonary arterial hypertension (PAH) and contra lateral pneumonectomy cases and that were unable to hold breath. ${ }^{[6]}$

Subsequently biopsy (bronchoscopic/trucut biopsy) or lobectomy specimens were then examined in pathology department for histopathological examination (HPE). The sections were stained with routine hematoxyline and eosin ( $\mathrm{H}$ and $\mathrm{E}$ ) stain, examined under light microscope. Immune-histochemistry was done in few cases, where necessary.

After data collection, they were tabulated and analysis was done with use of Med cal C software.

\section{Result and Analysis}

A total of 124 cases were included in the study where

Table 1: Demographic description of the study

\begin{tabular}{llcc}
\hline Subject & & Total No. & Percentage \\
\hline Age & & 4 & 3.22 \\
$<40$ yrs & & 16 & 12.9 \\
$40-49$ yrs & & 60 & 48.38 \\
$50-59$ yrs & & 40 & 32.28 \\
$60-69$ yrs & & 4 & 3.22 \\
70 yrs and & & & \\
above & & 80 & 64.51 \\
Sex & & 44 & 35.49 \\
Male & & & \\
Female & Right & 72 & 58.06 \\
Side of lesion & Left & 52 & 41.94 \\
& Smoker & 70 & 56.45 \\
History of & Non smoker & 54 & 43.55 \\
smoking & Benign & 6 & 4.83 \\
Provisional & 118 & 95.17 \\
diagnosis by CT & Malignant & & \\
scan/chest X ray & & 10 & 8.07 \\
Cytological & Benign & 114 & 91.93 \\
diagnosis & Malignant & 09 & 7.25 \\
Histological & Benign & 115 & 92.75 \\
diagnosis & Malignant & & \\
\hline & & & \\
\hline
\end{tabular}

we got both adequate FNAC smear and histopathology report of thoracic masses. Out of 124 cases, 80 cases (64.51\%) were male and $44(35.49 \%)$ were female. General demographic findings of the study and common disease pattern have been given in Table 1. In our study, cytological diagnosis showed maximum number of cases belonged to adenocarcinoma (48\%). The other diseases spectrum were squamous cell carcinoma $(21 \%)$, small cell carcinoma $(13 \%)$, carcinoid $(5 \%)$, nonspecific inflammation (4\%), large cell carcinoma $(2 \%)$, benign cystic lesion $(2 \%)$, granulomatous inflammation (2\%), dermoid (1\%), spindle cell neoplasm $(1 \%)$, and bronchiolo-alveolar carcinoma (1\%). There were four cases below 40 years of age. Majority of the cases were seen in fifth and sixth decade. Cytological examination showed that 114 cases were malignant and 10 cases were benign. Provisional diagnosis based as radiologic findings were 116 cases malignant and 8 cases benign. The diagnostic sensitivity of FNAC for bronchogenic carcinoma was $97 \%$ and specificity was $90 \%$, positive predictive value $99 \%$, negative predictive value $77 \%$. The diagnostic accuracy of CT guided FNAC was 95\% based on final HPE report.

In our study, a total of 114 malignant cases were detected by FNAC. Among the malignant lesions, most common carcinoma [Table 2] was adenocarcinoma 60 (52.63\%) followed by squamous cell carcinoma 26 (22.8\%), among malignant lesions. There was male preponderance in both adenocarcinoma $(2: 1)$ and squamous cell carcinoma $(5: 1)$. Smokers were most common (69\%) among squamous cell carcinoma cases.

FNAC showed that 114 cases were malignant and 10 cases were benign. Whereas HPE revealed 115 cases were malignant and 9 cases were benign. All cytological diagnoses were correlated with histological diagnoses [Table 3]. In few cases like carcinoid and spindle cell neoplasm (MPNST) we used Immuno histo-chemistry (IHC) i.e., chromogranin A, cytokeratin, and S-100 for confirmation of diagnosis. We found discordances regarding 3 benign and 3 malignant lesions.

\section{Benign lesion}

One lesion diagnosed as nonspecific inflammation by FNAC, finally that case was diagnosed by HPE as squamous cell carcinoma with secondary inflammation. The other two cases diagnosed by FNAC as benign cystic lesion and granulomatous inflammation turned out to be a Hydatid cyst and Aspergillosis [Figure 1a and b], respectively, after HPE.

\section{Malignant lesions}

Microscopically, most adenocarcinomas [Figure 1c and d], squamous cell carcinomas [Figure 2a], and small cell carcinomas [Figure 2b] showed classical cytomorphological features. In our study, we found six cases of carcinoid tumor [Figure 3], two cases of bronchiolo-alveolar carcinoma [Figure 2c and d], two cases of large cell anaplastic and one case of spindle cell neoplasm, which was finally diagnosed as 
Table 2: Distribution of malignant lesions according to sex and smoking habit

\begin{tabular}{|c|c|c|c|c|c|c|c|c|}
\hline \multirow[t]{2}{*}{ Malignant lesions (by FNAC) } & \multicolumn{2}{|c|}{ Male } & \multicolumn{2}{|c|}{ Female } & \multicolumn{2}{|c|}{ Smoker } & \multicolumn{2}{|c|}{ Non smoker } \\
\hline & No & $\%$ & No & $\%$ & No & $\%$ & No & $\%$ \\
\hline Adenocarcinoma $52.63 \%(n=60)$ & 40 & 67 & 20 & 33 & 32 & 53 & 28 & 47 \\
\hline Squamous cell carcinoma $22.8 \%(n=26)$ & 20 & 77 & 6 & 23 & 18 & 69 & 08 & 31 \\
\hline Small cell carcinoma $14.91 \%(n=17)$ & 9 & 53 & 8 & 47 & 10 & 58.8 & 07 & 41.2 \\
\hline Carcinoid $5.29 \%(n=6)$ & 4 & 67 & 2 & 33 & 4 & 67 & 2 & 33 \\
\hline Bronchiolo-alveolar carcinoma $1.75 \%(n=2)$ & 2 & 100 & Nil & & 2 & 100 & Nil & \\
\hline Large cell anaplastic carcinoma1.75\% $(n=2)$ & 2 & 100 & Nil & & 2 & 100 & Nil & \\
\hline Spindle cell neoplasm $0.87 \%(n=1)$ & 1 & 100 & Nil & & 1 & 100 & Nil & \\
\hline Total $=114$ & 78 & & 36 & & 69 & & 45 & \\
\hline
\end{tabular}
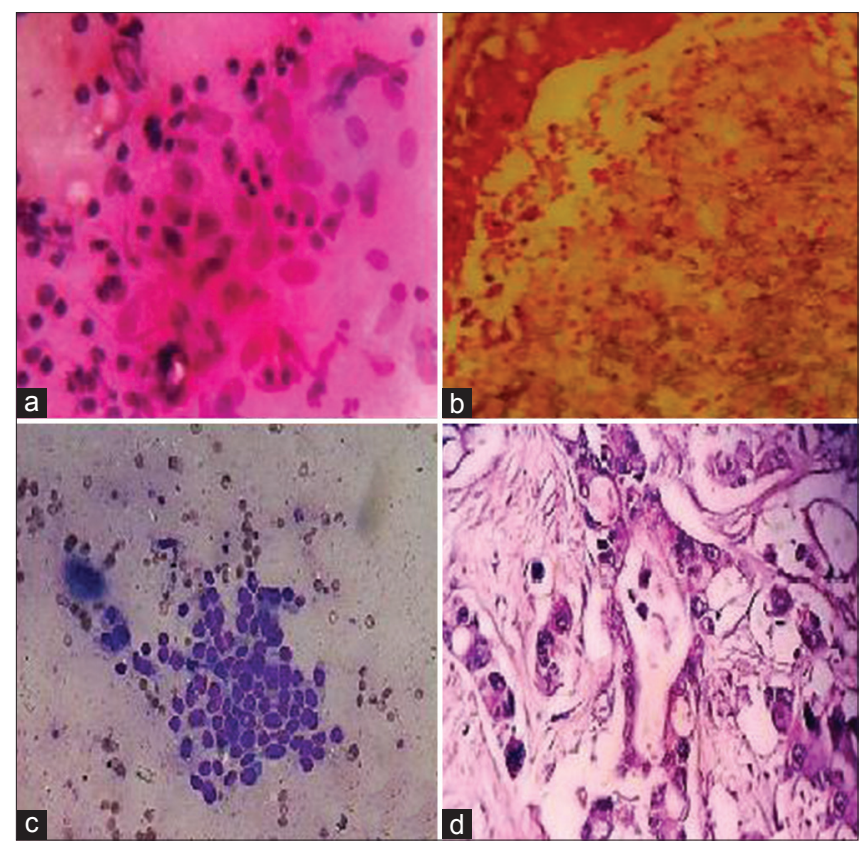

Figure 1: (a) Cytology smear showing ill formed granulomas (Leishman and Giemsa stain, $\times 400$ ), (b) Biopsy showing fungal colony of Aspergillosis (Hematoxyline and Eosin stain, $\times 400$ ), (c) Cytology smear showing cluster of epithelial cell arranged in acini. The cells have prominent nucleoli (Leishman and Giemsa stain, $\times 100$ ), (d) Histology section of lung mass showing well differentiated adenocarcinoma (Hematoxyline and Eosin stain, $\times 400$ )

malignant peripheral nerve sheath tumor (MPNST) by HPE as well as proved by immuno histochemistry (S-100 positive).

One case diagnosed as squamous cell carcinoma by FNAC but HPE showed chronic nonspecific inflammation with focal squamous metaplasia of lining respiratory epithelium. The other two cases FNAC marked as adenocarcinoma but HPE revealed those as poorly differentiated squamous cell carcinoma [Table 3].

In our study, HPE reports of pulmonary tumors were considered as final confirmatory report. So correlation was established between CT guided FNAC findings and provisional diagnosis by radiology by calculating weighted Kappa value. The weighted Kappa values were 0.816, 0.602, and $95 \%$ confidence interval were ( 0.64 to 0.99$)$ and $(0.39$ to 0.86 ) for CT guided FNAC reports and radiological diagnosis, respectively . It was found that $\mathrm{CT}$ guided FNAC diagnosis showed almost perfect agreement, whereas radiological

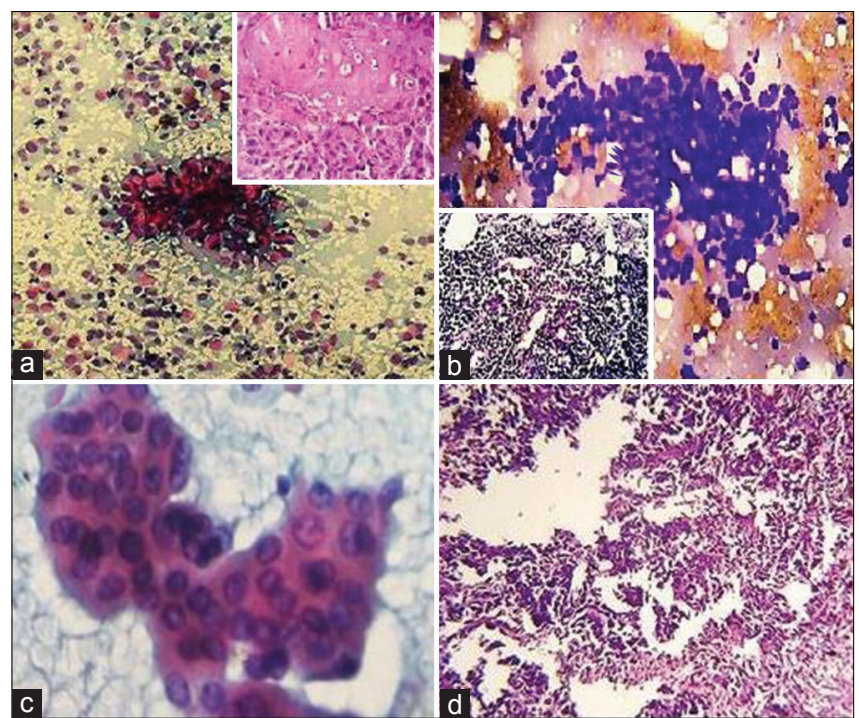

Figure 2: (a) Squamous cell carcinoma, Cytologic smear showing malignant squamous cells in clusters and dispersed singly (PAP stain $\times 400$ ), Histologic section showed (inset) malignant squamous cell in sheets with keratin pearl formation ((Hematoxyline and Eosin stain, $\times 400$ ), (b) Small cell carcinoma, Cytologic smears showing small sized cells in tight groups showing nuclear molding, coarse clomped chromatin ((Leishman and Giemsa stain, $\times 100)$, Histologic section showed (inset)-small cells with dark hyper chromatic nuclei, atypical mitosis, areas of necrosis,(Haematoxyline and Eosin stain, $\times 200$ ), (c) Bronchiolo-alveolar carcinoma, Smear showed round to oval uniform cells in monolayer sheet with high nuclear cytoplasmic ratio and intra nuclear groove. (Leishman and Giemsa stain, $\times 400$ ), (d) Bronchiolo-alveolar carcinoma, Histologic section showing lepidic pattern of growth ((Hematoxyline and Eosin stain, $\times 100)$

diagnosis showed moderate agreement with HPE diagnosis.

\section{Discussion}

FNAC is an accurate and safe method for evaluation of pulmonary mass. It enables categorization of malignant lesions in the vast majority of cases. It is also useful in diagnosis of dermoid. In our present study, 124 FNAC from pulmonary mass lesions were evaluated over a period of two years, all cases showed adequate cells in FNA smear as well as subsequently was performed irrespective of clinical diagnosis.

All cases enrolled were adults. The peak age of incidence (50-59 years) was the same as that documented in recent studies. ${ }^{[2,7]}$ Of these cases, $10(8.07 \%)$ were benign and $114(91.93 \%)$ were malignant as per FNAC findings. The reason may be due to increased incidence of malignancy 


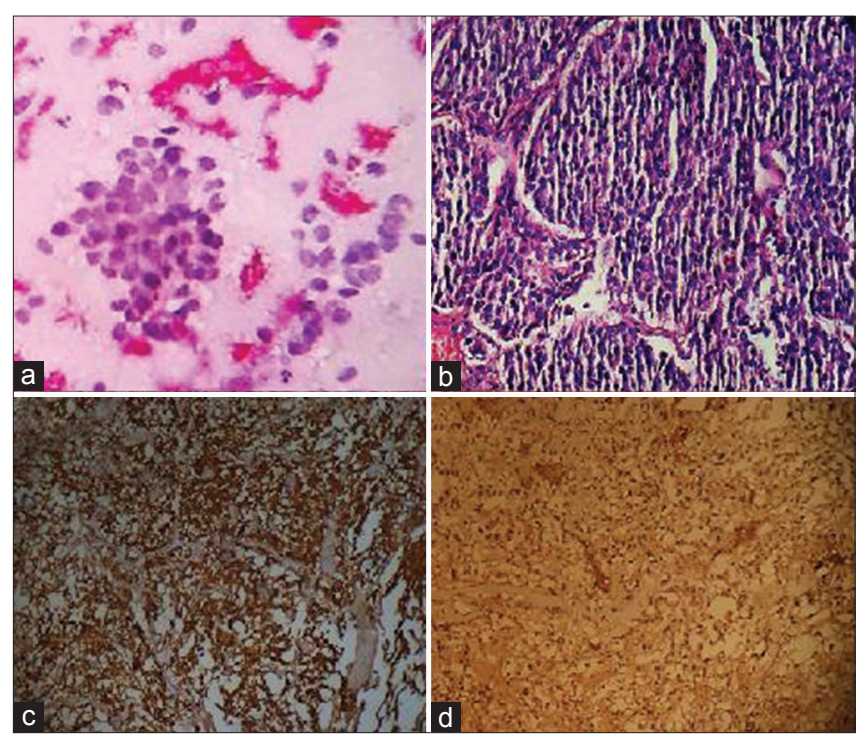

Figure 3: Carcinoid Tumor (a) Cytology smears showing monomorphic population of small round cells with stippled chromatin (Leishman and Giemsa stain, $\times 400$ ), (b) Histologic section showed uniform round cells forming nests and rosettes (Hematoxyline and Eosin stain, $\times 100$ ), (c) Immuno-histochemistry showed tumor cells positive for Chromogranin A positive, (d) Immuno-histochemistry showed tumor cells negative for Cytokeratin - negative

Table 3: Cytological and histopathological correlation

\begin{tabular}{|c|c|c|c|c|}
\hline $\begin{array}{l}\text { Cytological } \\
\text { diagnosis }\end{array}$ & Number & Correlation & Concordant & Discordant \\
\hline \multicolumn{5}{|l|}{ Benign } \\
\hline $\begin{array}{l}\text { Nonspecific } \\
\text { inflammation }\end{array}$ & 5 & 5 & 4 & 1 \\
\hline Dermoid & 1 & 1 & 1 & \\
\hline $\begin{array}{l}\text { Benign cystic } \\
\text { lesion }\end{array}$ & 2 & 2 & 1 & 1 \\
\hline $\begin{array}{l}\text { Granulomatous } \\
\text { inflammation }\end{array}$ & 2 & 2 & 1 & 1 \\
\hline \multicolumn{5}{|l|}{ Malignant } \\
\hline Carcinoma & 105 & 105 & 103 & 3 \\
\hline Carcinoid & 6 & 6 & 6 & \\
\hline Sarcoma & 1 & 1 & 1 & \\
\hline
\end{tabular}

in this age group as FNAC was mainly used for diagnosis for neoplasm. But HPE showed 9 (7.25\%) cases were benign and $115(92.75 \%)$ cases were malignant. The mean age in our study was 56.6 years, which is almost similar with (56.4 years) and (56.8 years) to studies conducted by Singh et al., ${ }^{[8]}$ and Saha et al., ${ }^{[7]}$ respectively. Power et al., ${ }^{[9]}$ also showed increased prevalence of thoracic neoplasm after the age of 50 years, with an average age of 54 years.

There was male preponderance $(64.51 \%)$ in our study compared to female. The percentage of male patients was a little higher in our series than in three recent studies i.e., $71.1 \%{ }^{[10]} 78.9 \%{ }^{[7]}$, and $80.6 \%{ }^{[11]}$, respectively.

In this study, lung tumors were located more on the right side than on the left. Among the patients, 56.45\% were active smokers. Cytological diagnosis was made in 124 out of 130 cases $(95.38 \%)$ and the high incidence of malignancy $(91.93 \%)$ was comparable with that found in other studies.
[7.11,12] The malignant cases formed largest category including carcinoma, neuroendocrine tumors, and sarcoma. Among all 114 cases of malignant pulmonary tumors, the most common ( $n=60 ; 52.63 \%$ ) was adenocarcinoma followed by $22.8 \%$ squamous cell carcinoma, and $14 \%$ small cell carcinoma. The incidence of adenocarcinoma was reported to be significantly higher than that of squamous cell carcinoma in recent studies by Tan et al., ${ }^{[10]}$ and Madan et al. ${ }^{[13]}$ Where as in certain other national and international studies, prevalence of squamous cell carcinoma was more than adenocarcinoma. ${ }^{[2,11,14-16]}$ In Our study we found $1 \%$ case of bronchiolo-alveolar carcinoma (BAC). There is change in concept regarding diagnosis of BAC. Travis et al., ${ }^{[17]}$ in 2011 recommended discontinuing the term 'BAC' (strong recommendation, low quality evidence). For small $(\leq 3 \mathrm{~cm})$ solitary adenocarcinoma with pure lepidic growth, they recommended the term adenocarcinoma in situ (AIS). For same size and same pattern of growth with small foci of invasion $\leq 0.5 \mathrm{~cm}$-a new concept of minimally invasive carcinoma (MIA) to be considered. But AIS or MIA should not be used in small biopsy or cytology specimen. So, BAC can be regarded as an adenocarcinoma with lepidic growth with or without invasion. But in our study, we followed the earlier classification of lung tumors and BAC was included one of the entities.

The efficacy of CT guided FNAC in the diagnosis of benign and malignant lesion was $67 \%$ versus $97 \%$. It is evident from the Table 2, adenocarcinoma, squamous cell carcinoma; small cell carcinoma, neuroendocrine carcinoma can be effectively diagnosed by cytology. A high degree of accuracy in cytological typing can be of great importance in those cases where no confirmatory histology is available. Maximum cases of lung malignancy were primary while only one case of represented as metastatic adenocarcinoma of prostate.

Though pneumothorax was most common complication of other studies, but we did not found any case in our study. The overall rate of complications $(2.4 \%)$ in our study was also remarkably less than other series where the range varied from 6 to $50 \% .^{[10,11]}$ In our study, mild hemorrhage from perilesional area in one case and chest pain in two cases was found and which could be managed conservatively.

In our study, CT guided FNAC showed almost perfect agreement with histological diagnosis in comparison to radiological opinion. So FNAC was found to be highly accurate (95\%) in diagnosis of lung mass as almost similar shown by previous studies. ${ }^{[13,14]}$ Hence CT guided FNAC diagnosis alone can be used with confidence to select treatment modalities and to avoid unnecessary surgeries in patients with lung malignancies.

\section{Conclusion}

CT guided FNAC is a simple, safe, and reliable procedure with high diagnostic accuracy for the diagnosis and sub typing of lung cancer. Though complications are rare, peri-lesional hemorrhage, and chest pain are occasionally encountered, but rarely require active management. 


\section{References}

1. Martin HE, Ellis EB. Biopsy by needle puncture and aspiration. Ann Surg 1930;92:169-81.

2. Shah S, Shukla K, Patel P. Role of needle aspiration cytology in diagnosis of lung tumors. A study of 100 cases. Indian J Pathol Microbiol 2007;50:56-8.

3. Ahmad M, Afzal S, Saeed W, Mubarik A, Saleem N, Khan SA, et al. Efficacy of bronchial wash cytology and its correlation with biopsy in lung tumours. J Pak Med Assoc 2004;54:13-6.

4. Mullan CP, Kelly BE, Ellis PK, Hughes S, Anderson N, Mc Cluggage WG. CT-guided fine-needle aspiration of lung nodules: Effect on outcome of using coaxial technique and immediate cytological evaluation. Ulster Med J 2004;73:32-6.

5. CoxJE, Chiles C, McManus CM, Aquino SL, Choplin RH. Transthoracic needle aspiration biopsy: Variables that affect risk of pneumothorax. Radiology 1999;212:165-8.

6. Hayes MM, Zhang DY, Brown W. Transthoracic fine-needle aspiration biopsy cytology of pulmonary neoplasms. Diagn Cytopathol 1994; 10:315-9.

7. Saha A, Kumar K, Choudhuri MK. Computed tomography-guided fine needle aspiration cytology of thoracic mass lesions: A study of 57 cases. J Cytol 2009;26:55-9.

8. Sing JP, Garg L, Setia V. Compared tomography (CT) guided transthoracic needle aspiration cytology in difficult thoracic mass lesions - not approachable by USG. Indian J Radiol Imaging 2004; 14:395-400.

9. Powers CN, Silverman JF, Geisenger KR, Frable WJ. Fine needle aspiration biopsy of the mediastinum a multi institutional analysis. Am J Clin Pathol 1996; 105: 168-73.

10. Tan KB, Thamboo TP, Wang SC, Nilsson B, Rajwanshi A, SaltoTellez M. Audit of transthoracic fine needle aspiration of the lung: Cytological sub classification of bronchogenic carcinomas and diagnosis of tuberculosis. Singapore Med J 2002;43:570-5.

11. Bandyopadhyay A, Laha R, Das TK, Sen S, Mangal S, Mitra PK. CT guided fine needle aspiration cytology of thoracic mass lesions: A prospective study of immediate cytological evaluation. Indian J Pathol Microbiol 2007;50:51-5.

12. Abrari A, Aziz M, Haq R. Cytology of lung tumors - study of 60 cases with emphasis on accuracy and problem areas. J Cyto 2003;20:79-81.

13. Madan M, Bannur. Evaluation of FNAC in lung diseases. Turk J Pathol 2010;26:1-6.

14. Jaya Shankar E, Pavani B, Chandra E, Reddy R, Srinivas M, Ashwin Shah. Computed tomography guided percutaneous thoracic: Fine needle aspiration cytology in lung and mediastinum. J Cytol Histol 2010;107:1-3

5. Basnet SB, Thapa GB, Shahi R, Shrestha M, Panth R. Computed tomography guided percutaneous transthoracic fine needle aspiration cytology in chest masses. JNMA J Nepal Med Assoc 2008;47:123-7.

16. Syed A, Shabab M, Uddin A. Computed tomography guided fine needle aspiration cytology of lung lesions: A study of 162 cases. J Chittagong Med Coll Teach Assoc 2009;20:50-2.

17. Travis WD, Brambilla E, Noguchi M, Nicholson AG, Geisinger K, Yatabe $\mathrm{Y}$, et al. International association for the study of lung cancer/American thoracic society/European respiratory society international multidisciplinary classification of lung adenocarcinoma. J Thorac Oncol 2011;6:244-85.

How to cite this article: Mondal SK, Nag D, Das R, Mandal PK, Biswas PK, Osta M. Computed tomogram guided fine-needle aspiration cytology of lung mass with histological correlation: A study in Eastern India. South Asian J Cancer 2013;2:14-8.

Source of Support: Nil. Conflict of Interest: None declared. 